\title{
Ion transmission to the focal plane of the Chandra X-ray Observatory
}

\author{
J.J. Kolodziejczaka, R.F. Elsnera, R.A. Austin, S.L. O’Della . \\ a NASA, Marshall Space Flight Center, SD50, Huntsville, AL 35812, USA \\ ${ }^{\mathrm{b}}$ USRA, Marshall Space Flight Center, SD50, Huntsville, AL 35812, USA
}

\begin{abstract}
The ACIS instrument on-board the Chandra X-ray Observatory (CXO) experienced pronounced degradation in spectrometric performance during the spacecraft's orbital activation and calibration phase. This damage was associated with a sharp increase in charge-transfer inefficiency combined with relatively constant dark current. Damage occurred only during passage through the earth's radiation belts, and only when ACIS remained in the focal plane during the passage. Subsequent measurements and analyses support the conjecture that the damaging radiation entered through the Observatory's High-Resolution Mirror Assembly (HRMA) aperture. A mechanism whereby low-energy magnetospheric protons and heavier ions are scattered through the HRMA and reach the focal plane with just enough energy to stop in the CCD's charge transfer channel provides a reasonably consistent explanation of all observed phenomena. In this paper, we shall describe analyses which support this conclusion. We simulated the mirror surfaces and various path elements using a standard ion transmission code to generate a bi-directional reflectance distribution function (BRDF). We then convolved the BRDF with the geometry using a ray-trace code. This paper presents damage estimates using measured proton fluences and ground measurements of ACIS-type CCD damage versus proton energy and compares them with observed on-orbit damage.
\end{abstract}

Keywords: Chandra, ACIS, proton damage, ion scattering, ion transmission, x-ray, HRMA, CCD, space radiation hazard.

\section{INTRODUCTION}

During the Chandra X-Ray Observatory's orbital activation and calibration phase, the front-illuminated ACIS (Advanced Charge-coupled device Imaging Spectrometer) CCDs sustained damage. ${ }^{1}$ This damage resulted in degradation of the spectral performance due to increased charge transfer inefficiency (CTI). The damage began after opening all contamination covers, and it occurred only during perigee passages through the earth's radiation belts on occasions when the ACIS was at focus and the transmission gratings were retracted. Ground measurements on identical CCDs showed similar damage characteristics after irradiation with relatively soft protons but no damage when irradiated with electrons. The damage was not consistent with that observed on previous missions resulting from more energetic protons because the change in dark current of the CXO devices was relatively small.

High energy proton damage is a well-understood phenomenon ${ }^{2}$ and the Chandra design protects ACIS from significant damage over its life. A search for sneak paths following discovery of the damage indicated that the design is sound with greater than $0.8 \mathrm{~g} / \mathrm{cm}^{2}$ of shielding everywhere when ACIS is at focus. This is far more than enough shielding to absorb the high flux of soft protons in the radiation belts.

It was apparent that the radiation was coming from the direction of the HRMA (High-Resolution Mirror Assembly) aperture because it didn't begin until the HRMA front contamination cover was opened and it did not occur when the High Energy Transmission Grating (HETG) was inserted. Therefore, it seemed likely that the mechanism that allowed the damaging radiation to reach ACIS was Rutherford scattering from the mirror surfaces. In this paper we present a quantitative analysis of this scenario to see if it provides a plausible explanation.

Because a strong correlation exists among the amount of damage, ACIS position with respect to HRMA focus, and the external radiation environment, it is possible to predict future damage with understanding of neither the specific damage mechanism nor the means by which the radiation reaches the focal plane. Determination of the damage mechanism is important because it is desirable to find ways of improving the detector spectral performance ${ }^{3}$, however the means by which 
the radiation reaches the focal plane is of little consequence as long as we have confidence in the protection afforded by our "safe position". A year of operation with no additional unexpected damage provides this confidence.

On the other hand, a more quantitative analytical demonstration that sufficient radiation to damage ACIS does indeed reach the focal plane would be useful in answering a number of important questions:

- $\quad$ Is the entire damage scenario plausible?

- $\quad$ Are the energies used in ground ACIS CCD irradiations appropriate?

- $\quad$ Do any unidentified sources of damage exist?

- What are appropriate design and risk criteria for other and future missions?

In the following sections we will describe the methods we have implemented to address these questions. We provide estimates of the ion transmission through the various components in the path to the sensitive region of the ACIS CCDs. We estimate the proton scattering distribution from the HRMA and gratings. Finally, we integrate these estimates along with estimates of the external environment during the times of the ACIS damage and the measured ACIS damage sensitivities to provide an overall estimate of expected damage resulting from protons through the HRMA aperture.

\section{ANALYSIS METHODS}

The analysis naturally divides into three main categories:

- the external radiation environment,

- the CTI damage susceptibility of the ACIS sensitive region,

- the scattering and transmission characteristics of all the elements in the path from the outside to the damage sites.

Details of the first two items are described elsewhere and are only briefly discussed here. The focus of this paper is the characteristics of the path elements.

The external radiation environment, as predicted by the AP8MAX model ${ }^{4}$, is shown in Figure 1(a). A crosscheck of these data indicate that they agree with the CRRESPRO model ${ }^{5}$. There is a factor of 5 difference between the high proton flux state and the low one. We include the high flux case for reference only because there is a strong consensus that the flux during the damaging belt passages was consistent with the quiet model to within a factor of 2 . More details on the radiation models are provided in References 6 and 7.

The ACIS CTI damage resulting from room temperature irradiation by soft protons is shown in Figure 1(b). Portions of an ACIS flight-like CCD were irradiated with a suitable dose at the various energies shown. Results show that the most damaging protons are those around 100-150 keV. Further details regarding these measurements are available in Reference 1.

(a)

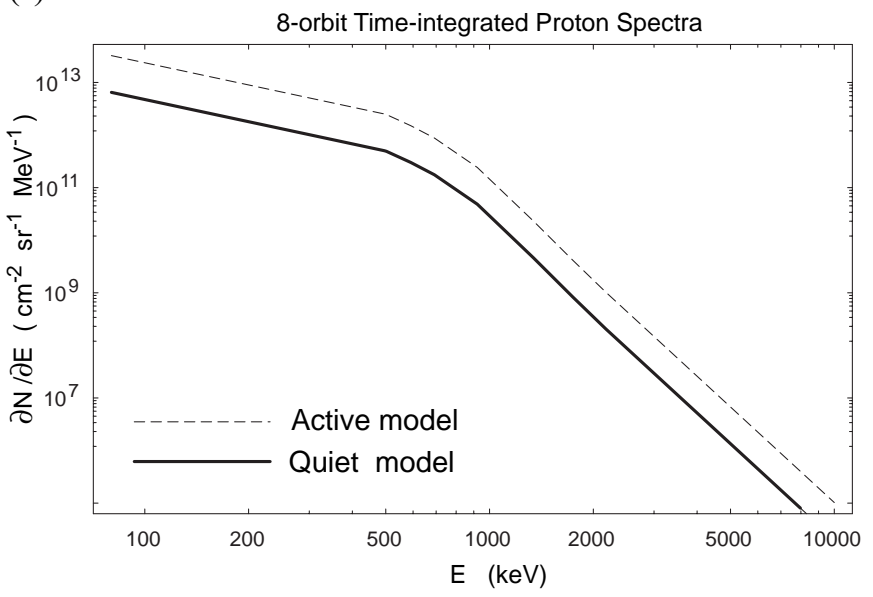

(b)

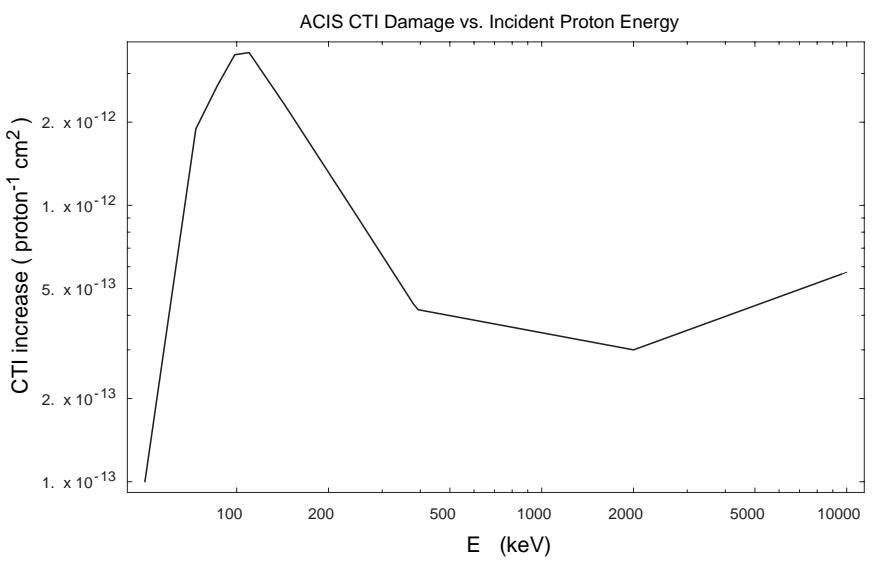

Figure 1. (a) shows 2 curves representing the proton environment integrated over the 8 damaging radiation-belt passages. The AP8MAX environment was used to obtain a proton spectrum and fluence and these were crosschecked with CRRESPRO. The CRRESPRO quiet-model prediction agreed with the AP8MAX model while the active model predicted higher flux by a factor of 5 . The quiet model curve is exactly the AP8MAX prediction while the active model curve is simply the AP8MAX result scaled by a factor of 5. (b) shows how ACIS CTI damage depends on incident proton energy. The curve is a log-log piece-wise interpolation of ground measurements of CTI damage. 
We used two tools to analyze scattering and transmission characteristic: An ion-implantation Monte-Carlo code called TRIM (Transport of Ions in Matter) ${ }^{8}$, and a custom ray trace code ${ }^{9}$ developed for study of HRMA x-ray response characteristics. A limited number of measurements of grazing-incidence proton scattering were made at Harvard University. We include these in the context of attempting to verify the TRIM results. They are briefly summarized in section 5 .

\section{ION PATH DESCRIPTION}

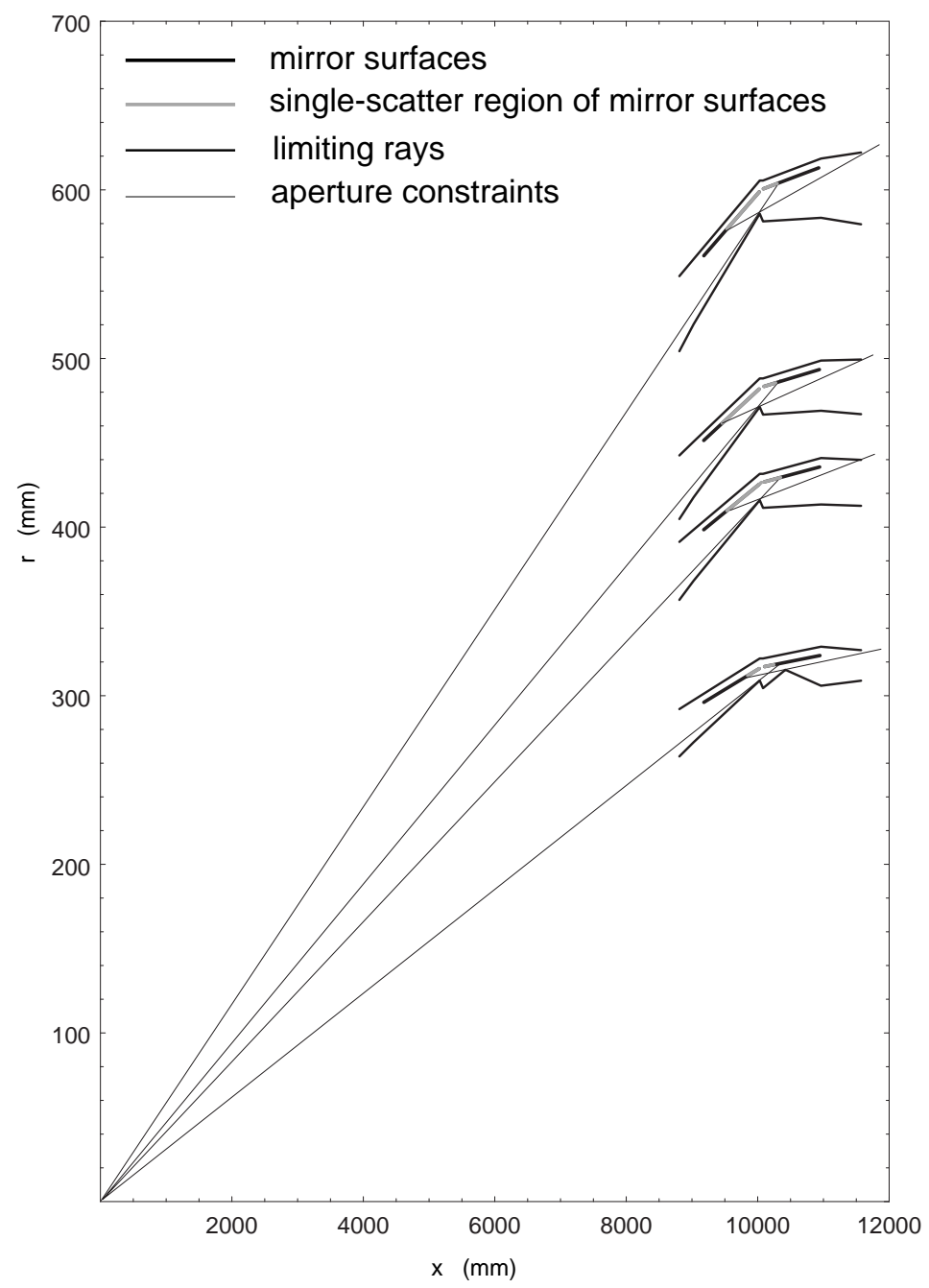

Figure 2. HRMA geometry indicating a region of the mirror surface visible by direct rays from both the outside and the HRMA focus. Ions from the external environment which impinge upon this surface may reach the focal plane through a single scatter
The path that an ion must follow to reach the sensitive region of an ACIS CCD requires transmission through or scattering from the following elements:

- HRMA precollimator

- Parabolic Shells

- Central apertures

- Hyperbolic Shells

- HRMA postcollimator

- Grating substrate

- $\quad$ ACIS optical blocking filter (OBF)

- $\quad$ ACIS chips

Fig. 2 shows the geometric configuration of the first 5 elements. It is apparent in from this Figure that a significant portion of the mirror surfaces is visible to both the telescope focal plane and the outside environment. This region is of particular interest since it is a region whereby ions may reach the HRMA focus after only a single scattering interaction.

The HRMA reflecting surfaces consist of three layers-a thick (1.6-2.4 $\mathrm{cm}$ depending on diameter) Zerodur ${ }^{\mathrm{TM}}$ substrate coated with $10 \mathrm{~nm}$ of Chromium followed by $32.5 \mathrm{~nm}$ of Iridium. Some hydrocarbon molecular contamination is likely in the thickness range 1-4 $\mathrm{nm}$. The reflecting surfaces are located between 9.2 and $10.9 \mathrm{~m}$ from the focus as measured along the optical axis.

The HETG is located $8.6 \mathrm{~m}$ from focus and consists of many individual grating facets. The facets used for the inner 2 HRMA shells (HEG) have a $1000 \mathrm{~nm}$ polyimide substrate while the substrates for facets on the outer 2 shells (MEG) are $500 \mathrm{~nm}$ thick. Additionally, each substrate has a $5 \mathrm{~nm} \mathrm{Cr}+60 \mathrm{~nm}$ Au coating. The geometric area is reduced by a factor of 2-2.5 by the grating bars and support structure. The HETG was not in the optical path during the damaging radiation belt passages.

The ACIS Optical Blocking Filter (OBF) is located $16 \mathrm{~mm}$ from focus and has 2 distinct regions covering the different parts of the detector. 6 CCDs designated as the S-Array are protected by $200 \mathrm{~nm}$ of polyimide coated with aluminum:- $90 \mathrm{~nm}$ on one side and $30 \mathrm{~nm}$ on the other. The other 4 CCDs, designated as the I-array are protected by the same thickiness of polyimide coated with $130 \mathrm{~nm}$ and $30 \mathrm{~nm}$ of aluminum on the two sides.

We model the CCD chips as several distinct layers. The front illuminated devices have a gate structure overlaying the sensitive charge transfer region. For the structure, we take $300 \mathrm{~nm} \mathrm{Si}$ over $300 \mathrm{~nm} \mathrm{SiO}{ }_{2}$ over $40 \mathrm{~nm} \mathrm{Si}_{3} \mathrm{~N}_{4}$ over the $\mathrm{Si}$ substrate. 


\section{TRANSMISSION THROUGH OBF AND GATE STRUCTURE}

We used TRIM software to model the transmission through the ACIS OBF and the CCD gate structure as a means to obtain an estimate of the energy of the damaging radiation. Since the charge transfer region is directly below the gate structure, we expect that the any CTI damage results primarily from ions with just enough energy to reach this region but not so much as to pass through the region.

Figure 3(a) shows the front-illuminated chip simulation results for energies in the range from $25 \mathrm{keV}$ to $1 \mathrm{MeV}$ for the combined $\mathrm{OBF}$ and gate structure indicating that transmission changes from $0 \%$ to $100 \%$ over the energy range between 70 and $90 \mathrm{keV}$. We show a plot of transmission through the $\mathrm{OBF}$ alone in Fig 3(b). This indicates that 20 to $30 \mathrm{keV}$ is required to pass through the $\mathrm{OBF}$. These values are consistent with the low-energy damage threshold observed in the ground-irradiated CCDs. Similar simulations of singly-ionized oxygen indicate that $\sim 400 \mathrm{keV}$ is needed to reach the damage-sensitive charge transfer region in that case.

The spectrum of the external environment must be modified to account for energy loss through the OBF before applying the results of the ground ACIS measurements since these were not performed with an OBF. This was done trivially by adjusting the energy scale in Fig 1(b) downward by $26 \mathrm{keV}$ before using this data in the calculations described below.

(a)

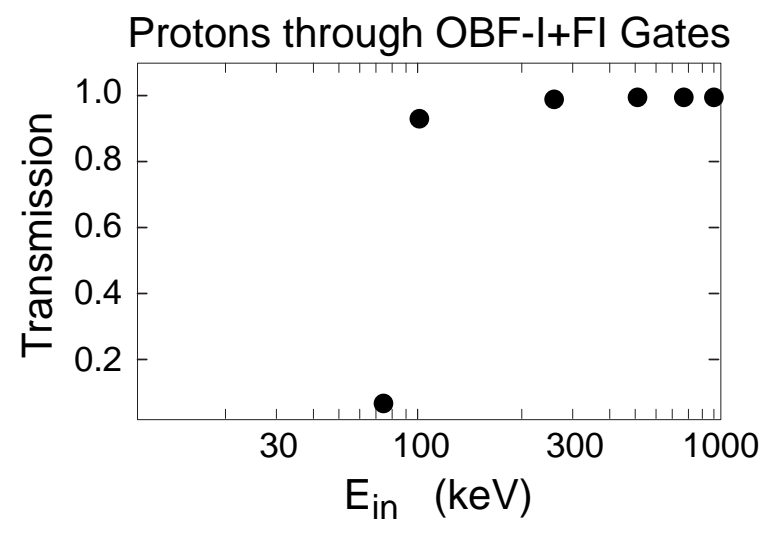

(b)

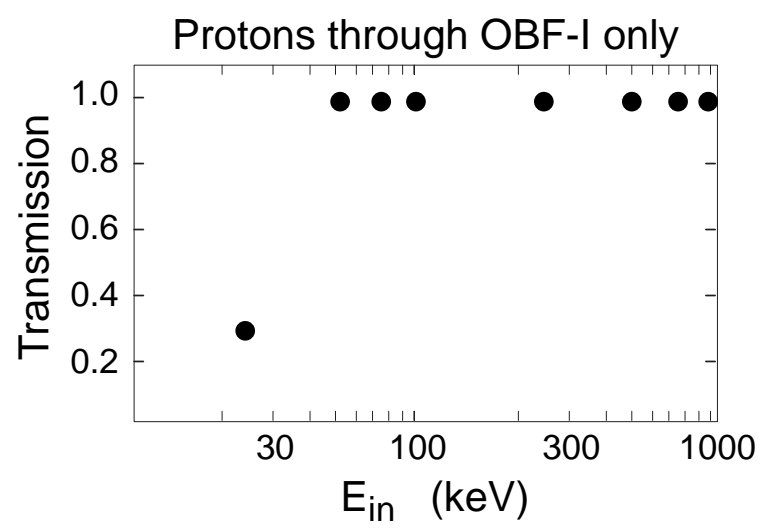

Figure 3. Results of TRIM simulations of proton transmission through (a) the combined ACIS-I OBF and frontilluminated chip gate structure and (b) the ACIS-I OBF alone. (a) indicates that an incident proton must possess at least $80-90 \mathrm{keV}$ in order to reach the sensitive charge-transfer region of the FI CCDs. (b) shows that the OBF effectively absorbs $26 \mathrm{keV}$ of energy from soft protons. Further simulations indicate that this is relatively insensitive to energy up to about $1 \mathrm{MeV}$.

\section{SCATTERING FROM HRMA SURFACES}

Some ions passing through the HRMA aperture impinge on the iridium surfaces and are scattered. In the regions of the surface highlighted in Fig. 2, some fraction of these ions will reach the HRMA focus after a single small-angle scatter. In addition, but less significantly, numerous multiple scattering paths exist, including scattering from the outside surfaces of the HRMA shells.

We use a ray trace code to evaluate all possible paths to the focal plane. The code was modified to allow the input of a bidirectional reflectance distribution function (BRDF), $\Psi\left(\theta_{\text {in }}, \mathrm{E}, \theta_{\text {out }}, \phi_{\text {out }}\right)$. The BRDF gives the probability distribution in 2 angular dimensions for a scatter out of the surface as a function of the incident angle and energy. The ray trace uses MonteCarlo methods to generate a scattered ray for every incident ray, based on this probability function.

We derived a BRDF for protons using results from TRIM. A set of TRIM simulations was performed with variable incident angle and incident proton energy over a matrix including 8 energies, $\{25,50,75,100,250,500,750,1000 \mathrm{keV}\}$ and 4 incident angles, $\left\{0.74^{\circ}, 0.79^{\circ}, 0.80^{\circ}, 0.87^{\circ}\right\}$. The TRIM data was parameterized in terms of 4 variables as a function of the incident angle, $\theta_{\mathrm{in},}$ and energy, E, as follows, 


$$
\Psi\left(\theta_{\text {in }}, \mathrm{E}, \theta_{\text {out }}, \phi_{\text {out }}\right)=\mathrm{R}\left(\theta_{\text {in }}, \mathrm{E}\right) \frac{\mathrm{e}^{\frac{-\left(\theta_{\text {out }}-\theta_{\text {out }}\right)^{2}}{2 \sigma_{i}^{2}}} \mathrm{e}^{\frac{-\left(\phi_{\text {out }}-\phi_{\text {out }}\right)^{2}}{2 \sigma_{o}^{2}}}}{\sqrt{2 \pi} \sigma_{o} \int_{0}^{\pi} \mathrm{e}^{\frac{-\left(\theta_{\text {out }}-\theta_{\text {out }}\right)^{2}}{2 \sigma_{i}^{2}}} d \theta_{\text {out }}}
$$

where $\sigma_{\mathrm{o}}$ is the out-of-plane gaussian width, $\sigma_{\mathrm{I}}$, is the in-plane gaussian width, $\theta_{\text {out }}$ is the peak in-plane exit angle, and $R$ is the total fraction scattered out of the surface. This parameterization is an idealization of the TRIM results, which tend to produce an extended tail, but the fraction of flux in that tail is $\sim 10 \%$ or less and we choose not to include it in the parameterized model. Figure 4 shows the values of these parameters as a function of energy and incident angle. According to the TRIM results, energy loss is negligible in scattering out of the surface, so the energy of the incident and scattered rays is taken to be the same.
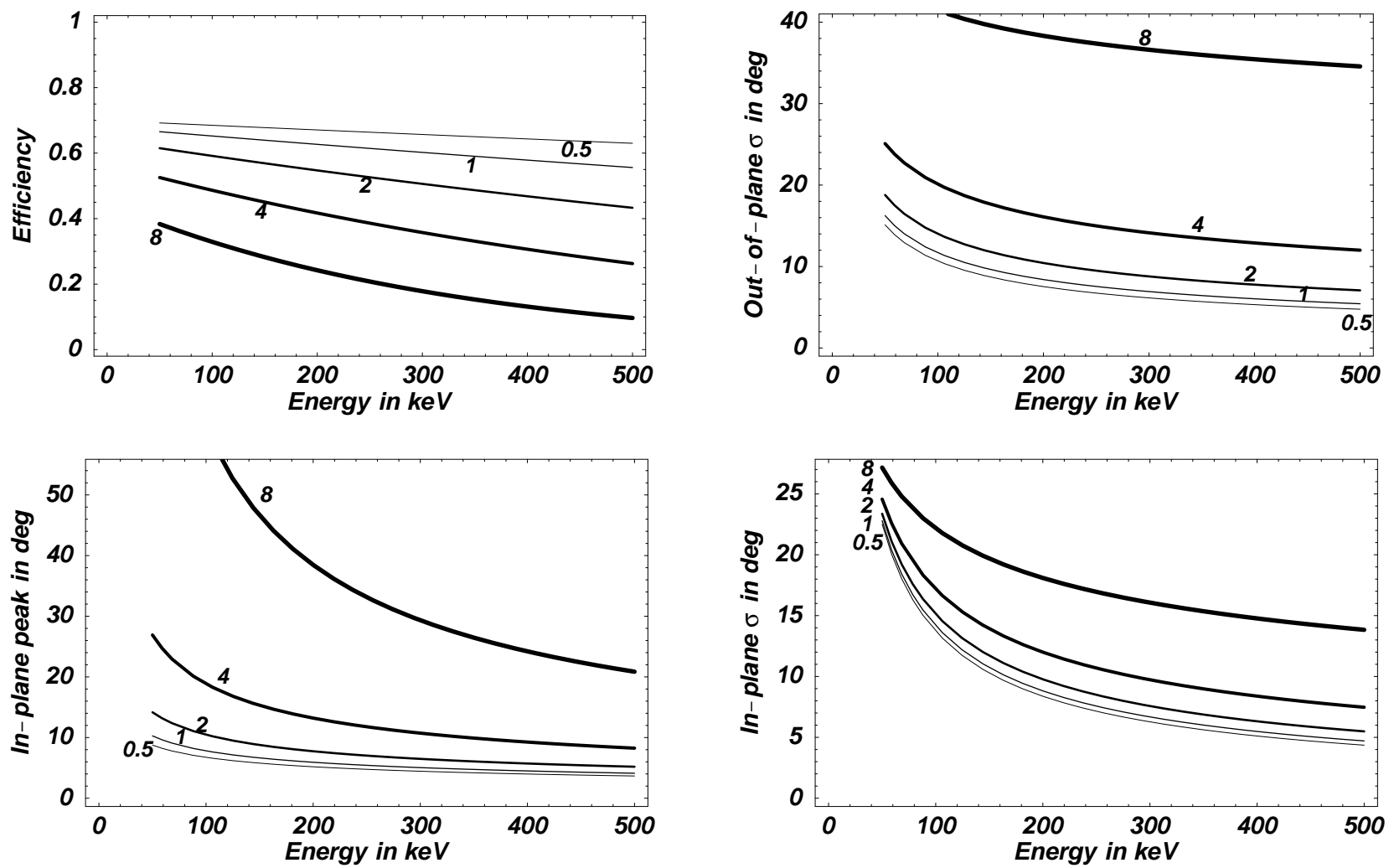

Figure 4. Model parameters were derived from TRIM simulations. The upper-left plot shows the scattering efficiency, $\mathbf{R}$. The lower-left plot shows the peak in-plane scattering angle, $\theta_{\text {out }}$. The upper-right plot shows the peak out-of-plane scattering angle, $\sigma_{0}$. The lower-right plot shows the peak in-plane scattering angle, $\sigma_{i}$. Each plot has several curves representing the parameter as a function of the incident proton energy, $E$, for several values of the incident angle, $\theta_{\text {in }}$.

A limited number of measurements of proton scattering at grazing incidence were performed at Harvard University ${ }^{10}$. Table 1 lists a summary of the approximate results compared with our TRIM parameterization. Agreement is poor, and the general indication is that both the in-plane scattering angle relative to the surface and the total fraction of scattering out of the surface are much smaller than either the TRIM model or that required to produce significant flux at the focal plane. The limited number of points prevents us from attempting to produce a BRDF from the data. Qualitatively, the lower scattering efficiency and smaller exit angle would make it more difficult for scattered flux to reach the HRMA focus and thus imply a lower damage contribution due to this mechanism. The difficulty of performing these measurements and the possibility of a systematic difference between the Harvard tests and the on-orbit situation cloud interpretation of these results. 


\begin{tabular}{|l|c|c|c|c|c|}
\hline & $\theta_{\text {in }}$ & $\mathrm{R}$ & $\theta_{\text {out } 0}$ & $\sigma_{\mathrm{i}}$ & $\sigma_{\mathrm{o}}$ \\
\hline \multirow{2}{*}{$\begin{array}{l}\text { Harvard } \\
\text { Measurements }\end{array}$} & $0.7^{\circ}$ & $13 \%$ & $0.1^{\circ}$ & $\sim 0.15^{\circ}$ & $\sim 0.8^{\circ}$ \\
\hline \multirow{2}{*}{$\begin{array}{l}\text { TRIM } \\
\text { Simulations }\end{array}$} & $1.0^{\circ}$ & $8 \%$ & $0.5^{\circ}$ & $\sim 0.5^{\circ}$ & $\sim 0.8^{\circ}$ \\
\cline { 2 - 6 } & $0.7^{\circ}$ & $66 \%$ & $4^{\circ}$ & $6^{\circ}$ & $5.5^{\circ}$ \\
\hline
\end{tabular}

Table 1. A comparison of Harvard measurements with TRIM simulation for a proton energy of $312.8 \mathrm{keV}$ indicates poor agreement. The measured scattering efficiency was much lower and the scattered beam was much more collimated and close to the surface than those values predicted by TRIM.

We show the results of the ray trace in Fig. 5. Fig. 5(a) shows a comparison of the single-scatter to multiple-scatter contribution to the focal plane and indicates, as expected, that singly-scattered ions are the dominant contributor to the focal plane flux, resulting in over $90 \%$ of the flux at the detector. Fig. 5(b) shows the relative contributions of the various shells, the innermost shell is disproportionately smaller because of an additional aperture baffle, shown in Figure 2, which reduces the amount of single scattering surface area. The units of the acceptance are steradians, but this should be interpreted as external $\mathrm{cm}^{2}$ sr per focal plane $\mathrm{cm}^{2}$ in converting external flux in units of protons $/ \mathrm{cm}^{2} / \mathrm{sr}$ to focal-plane dose in protons $/ \mathrm{cm}^{2}$.

a)

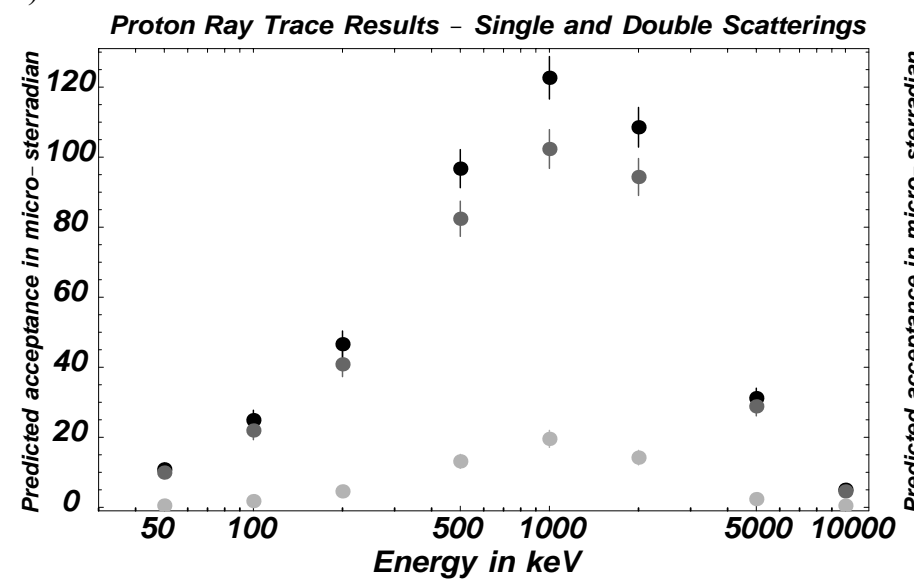

b)

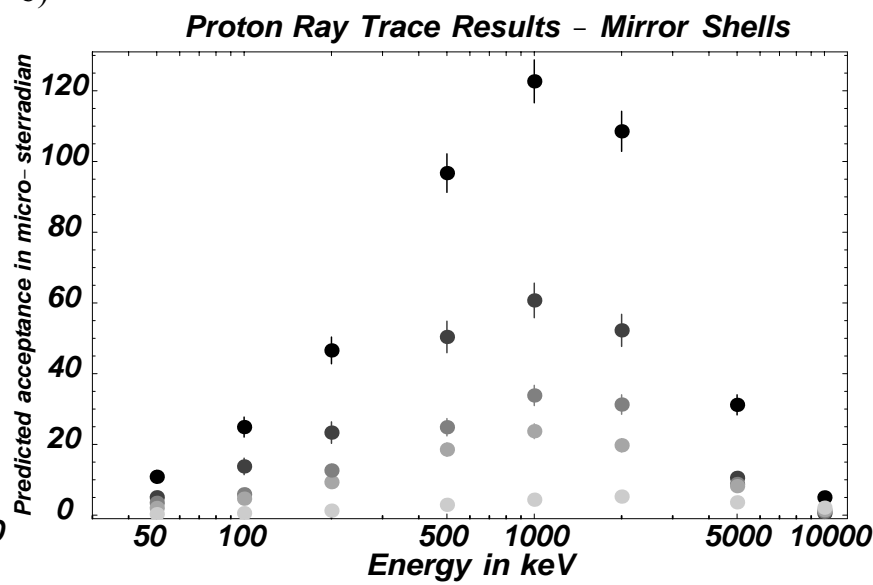

Figure 5. Proton acceptance at HRMA focus derived from ray-trace results using the scattering distribution parameters shown in Figure 4. (a) compares the relative contributions of singly (dark gray) vs. doubly (light gray) scattered rays. (b) compares the relative contributions of the four HRMA shells with colors going from light gray to dark gray indicating shells going from inner to outer. In both plots, the black points represent the total predicted acceptance.

\section{SCATTERING FROM HETG SUBSTRATE}

There were a small number of radiation belt passages in which ACIS remained at the HRMA focus and yet no additional damage was incurred. During these passages the HETG was inserted. We performed TRIM simulations which included the grating facets described in Section 3. The low-energy cutoff for the combined transmission of protons through the MEG substrate, OBF and front-illuminated ACIS gate structure is $140 \mathrm{keV}$. A similar value for the HEG facets is $190 \mathrm{keV}$. Based on this information, the energy range of damaging protons must lie in a narrow band between 80 and $140 \mathrm{keV}$. However, energy loss through the material is not the only mechanism by which the grating facets cause a reduced flux at the HRMA focus.

Additional reduction in flux at the HRMA focus arises as a result of dilution of the scattered beam. The cone of rays exiting each HRMA shell is collimated in the radial direction by the exit aperture at a half-angle of approximately $1^{\circ}$. Scattering in the grating facets tends to increase the angular extent of the exit beam and thereby dilute the flux at focus. Since the gratings are far from the HRMA focus, we can estimate the magnitude of this dilution factor, $k_{d}$, by the following equation, 


$$
k_{d}=\frac{\omega_{i}}{\sqrt{\omega_{i}^{2}+\omega_{s}^{2}}} \times \frac{\omega_{o}}{\sqrt{\omega_{o}^{2}+\omega_{s}^{2}}}
$$

where $\omega_{i}, \omega_{o}$ are in-plane and out-of-plane beam widths at the HRMA exit, and $\omega_{s}$ is the scattered beam width out of the grating from a perfectly collimated incident beam. Based on the scattering distributions obtained from the TRIM simulations described above, we estimate the $k_{d}=0.3$ at $200 \mathrm{keV}$, and $k_{d}=0.6$ at $500 \mathrm{keV}$. Combining the effects of grating substrate transmission, $50 \%$ grating bar opacity, and scattered flux dilution, it is not surprising that no damage was detected with the HETG inserted.

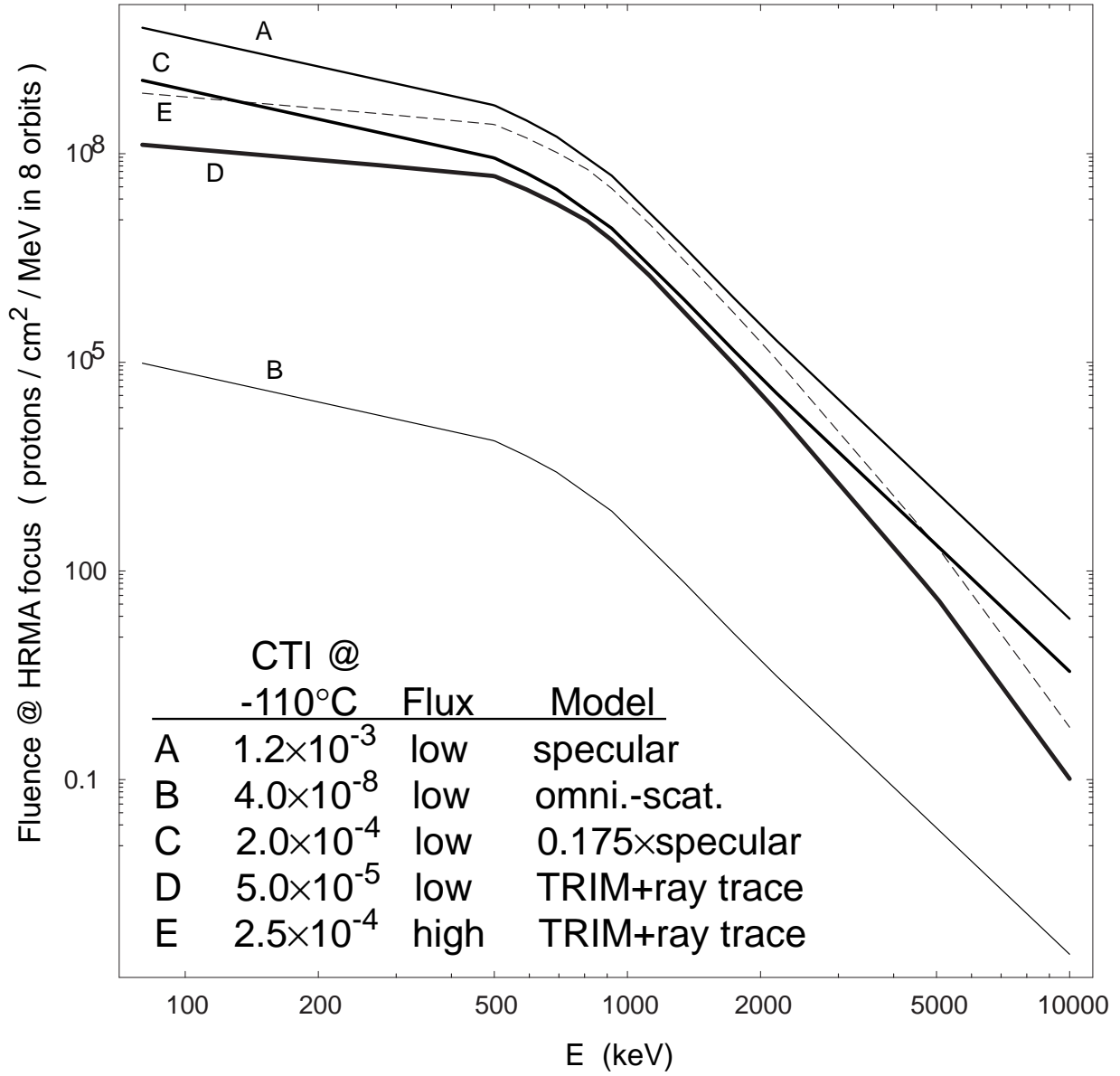

Figure 6. Various estimates of the proton fluence observed by the ACIS instrument during the 8 exposed radiation belt passages are shown. Curves $A, B, C$, and $D$ assume the external environment corresponds to the lower curve in Fig. 1(a). Curve $E$ assumes the upper curve in Fig. 1(a). Curve A represents the hypothetical case of $100 \%$ specularly reflective protons. Curve $B$ represents the case of uniform omni-directional scattering. Curve $\mathrm{C}$ represents the case of sufficient specular scattering to produce the observed damage. Curves $D$ and $E$ represent low and high proton flux cases for a total HRMA acceptance shown in Fig. 5.
The HETG instrument team plans to irradiate grating facets with soft protons to look for long-term performance effects. At that time we also plan to measure the transmission and dilution.

\section{PREDICTED ACIS DAMAGE}

The total expected ACIS damage is the integral over all energies of the product of focal-plane fluence, $f(\mathrm{E})$, and ACIS damage per unit fluence $\Delta \mathrm{CTI}(\mathrm{E})$. The focal plane fluence is the product of the acceptance, $\alpha(\mathrm{E})$ and external proton fluence $x(\mathrm{E})$. The fluence of interest here is the integral over the time intervals of the 8 exposed radiation belt passages of the proton flux distribution $p(\mathrm{E})$ as shown in Fig. 1(a). For the purposes of acquiring a sense of the bounds of the problem, we consider several cases illustrated in Fig. 6. Fig. 1(b) shows the damage per (proton $/ \mathrm{cm}^{2}$ ) used in these calculations based on ground measurements of irradiated CCDs.

We derived the top curve (A) in Fig. 6 from the simple assumption that the protons behave exactly the same as low-energy $\mathrm{x}$-rays, reflecting specularly with nearly $100 \%$ efficiency. In this case the acceptance is simply the product of the effective area, $\sim 1000 \mathrm{~cm}^{2}$, and the focal plane acceptance per unit area $10^{-6} \mathrm{sr} / \mathrm{cm}^{2}$. The latter is derived from fact that one $\mathrm{cm}^{2}$ at the focal plane subtends a solid angle of $10^{-6} \mathrm{sr}$ at a radius equal to the focal length of the telescope. The curve results from letting $\alpha=10^{-3}$ for all energies, and calculating, $x(\mathrm{E}) \times \alpha$. We derive a final ACIS CTI of $1.2 \times 10^{-3}$ at $-110^{\circ} \mathrm{C}$ using $\mathrm{CTI}=\int x(\mathrm{E}) \times \alpha(\mathrm{E}) \times \Delta \mathrm{CTI}(\mathrm{E}) d \mathrm{E}$ for this "worst case" scenario.

The trivial best case would be no scattering or reflectivity as all, but a more realistic best case is if a proton has an equal probability of scattering in any direction. In other words the scattering distribution function is uniform over $4 \pi$. A 
calculation involving integration of this scattering function over all possible angles and the entire HRMA aperture leads to an energy independent acceptance of $3 \times 10^{-8}$ sr. This case is illustrated in the lower-most curve (B) in Fig. 6. The resulting ACIS CTI increase in this case is $4 \times 10^{-8}$.

While these two values are not truly the most extreme values one can derive in an ad hoc manner, they do represent interesting benchmarks for comparison with more realistic models. One can scale the specular "worst case" curve to derive curve $\mathrm{C}$ which would lead to the exact amount of damage that was observed by ACIS. This corresponds to an acceptance of $1.6 \times 10^{-4} \mathrm{sr}$. To illustrate the scale, this is the equivalent of the focal-plane acceptance of a $12-\mathrm{cm}$ square hole $11 \mathrm{~m}$ away (approximate distance to the HRMA entrance aperture). Alternatively, it corresponds to $17 \%$ of the specular case, which is the value that would result from $41 \%$ reflectivity from each surface.

The curves representing the TRIM BRDF ray trace are calculated showing the low proton flux case (D) and the high flux case (E). For both of these cases, we used the total acceptance shown in Fig. 5. The integrated CTI increase for these two cases are $5 \times 10^{-5}$ and $2.5 \times 10^{-4}$, respectively. The low proton flux case comes very near the curve representing the observed damage at around $1 \mathrm{MeV}$, but the decline in acceptance at low energies leads to smaller predicted damage.

Fig. 7 shows the fraction of damage caused by protons above a given energy comparing the TRIM BRDF result to the curve corresponding to the real damage. These results are a further indication that soft protons between 100 and $200 \mathrm{keV}$ are the principal source of the damage. The result also shows that this conclusion is quite independent of scattering model.

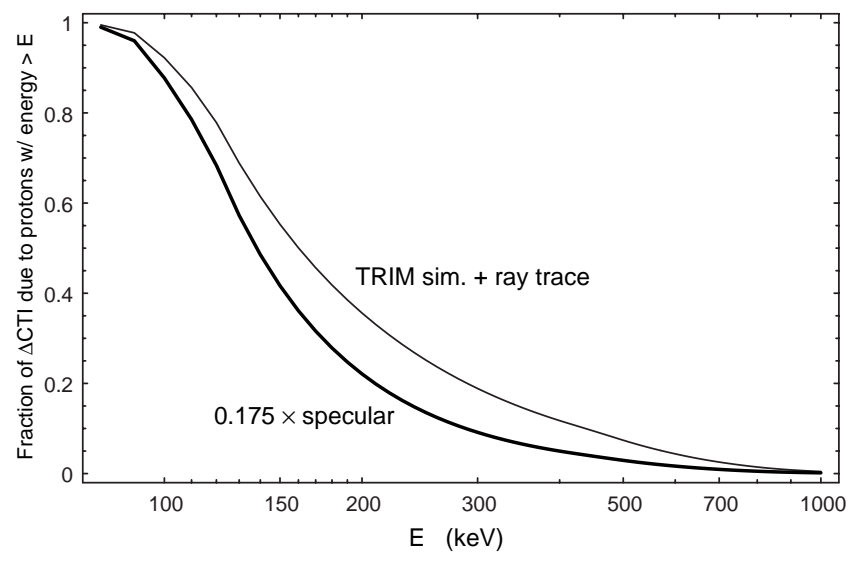

Figure 7. Comparison of fractional CTI damage by all protons above a given energy between the the TRIM BRDF ray trace model and the simple specular case. The conclusion that soft protons in the 100-200 $\mathrm{keV}$ range are the most damaging is not model dependent.

\section{CONCLUSIONS}

The results presented support the plausibility of an ACIS damage scenario where low-energy protons are scattered from the HRMA surfaces. It is only marginally plausible that this is the only source of damage. This position is justified based on the fact that observed damage is a factor of 4 higher than predicted for low proton flux. Because we have high confidence that the proton flux was relatively low during the damaging radiation belt passages, it appears that the model underestimates the damage by a factor of 3-4, accounting for flux uncertainties. We cannot, therefore, rule out the possibility that this scattered proton induced damage is augmented by some other mechanisms.

Some possible augmenting processes include the following either individually or in combination:

- damage by heavier ions, such as He or $\mathrm{O}$ by similar scattering processes. While the flux of these species are typically much lower than protons at energies of interest, it is conceivable that their capacity to produce damage is much higher.

- charging on the HRMA surfaces may lead to a change in electrostatic potential while passing through the radiation belts. This potential could change the distribution of incident angles and significantly modify the overall HRMA transmission.

- as-of-yet unidentified paths of ion scattering or direct transmission to the focal plane

- differences in the way damage occurred on-orbit versus ground irradiations could lead to an underestimation of ACIS damage susceptibility. On-orbit the chips were cooled $\left(-100^{\circ} \mathrm{C}\right)$ when irradiated then warmed to $20^{\circ} \mathrm{C}$, then re-cooled to $-110^{\circ} \mathrm{C}$. On the ground, they were irradiated warm before being cooled to test performance.

Alternatively, it is possible that our estimates are too low because of inaccuracies in the scattering distribution and BRDF derived from TRIM studies. For example, if we require the scattering distribution to remain constant at the $1-\mathrm{MeV}$ parameter values for all lower energies, we would obtain good agreement between observed and predicted values. Limitations of TRIM in accounting for small angle scattering processes could lead to scattering distributions which are too 
broad, and thereby underestimate the focal plane flux. The simulations are somewhat sensitive to the thickness of the molecular contamination layer as well, but we have not yet quantified these sensitivities. Measurements described in Section 5 support the hypothesis that the scattering distribution is not as broad as TRIM predicts, however, this is somewhat offset by the fact that the measured reflectivities are lower.

Finally, given that ACIS has not seen significant damage in more than a year, none of the identified factors could lead to unexpected damage as long as current safing procedures are maintained for the life of the mission. This implies high confidence that little or no unexpected future damage will occur.

\section{ACKNOWLEDGMENTS}

The authors gratefully acknowledge the efforts of Mark Bautz (MIT) and Gordon Garmire (Penn State), as well as Jene Golovchenko (Harvard) for providing unpublished information included herein.

\section{REFERENCES}

- $\quad$ G. Prigozhin, S. Kissel, M. Bautz, C. Grant, B. LaMarr, R. Foster, G. Ricker, G. Garmire, "Radiation damage in the Chandra X-ray CCDs", Proc. SPIE, 4012, pp. 720-729. 2000.

- K. Gendreau, M. Bautz, G. Ricker, "Proton damage in X-ray CCDs for space applications: Ground evaluation techniques and effects on flight performance", Nuclear Instruments and Methods in Physics Research, A, 335, pp. 318327, 1993.

- L. Townsley, P. Broos, G. Garmire, J. Nousek, "Mitigating Charge Transfer Inefficiency in the Chandra X-Ray Observatory Advanced CCS Imaging Spectrometer", Ap. J. , 534, pp. L139-L142, 2000.

- D. Saywer, J. Vette, "Trapped Particle Environment for Solar Maximum and Solar Minimum (AP8)", NSSDC Report 76-06, 1976.

- M. Gussenhoven, E. Mullen, M. Violet, C. Hein, J. Bass, D. Madden, "CRRES High Energy Proton Flux Maps", IEEE Trans. Nucl. Sci., 40, pp. 1450-1457, 1993.

- S. O’Dell, M. Bautz, W. Blackwell, Y. Butt, R. Cameron, R. Elsner, M. Gussenhoven, J. Kolodziejczak, J. Minow, D. Swartz, A. Tennant, S. Virani, K. Warren, "Radiation environment of the Chandra X-ray Observatory" Proc. SPIE to be published.

- W. Blackwell, J. Minow, K. Warren, R. Suggs, S. O’Dell, D. Swartz, A. Tennant, S. Virani, "Modeling the Chandra space environment" Proc. SPIE to be published.

- $\quad$ J. Ziegler, J. Beirsack, U. Littmark, The Stopping and Range of Ions in Solids, Pergamon Press, New York, 1996.

- R. Elsner, M. Joy, S. O’Dell, B. Ramsey, M. Weisskopf, "Ground-to-orbit transfer of the AXAF-I flux scale: in-situ contamination monitoring of x-ray telescopes”, Proc. SPIE, 2279, pp. 332-342, 1994.

- J. Pegg, M. Thrasher, M. Quirk, "Proton Reflectivity Off X-ray Mirrors at Glancing Angles", privately transmitted Harvard undergraduate project report, 2000. 